\title{
Dynamic Characterization of an Inflatable Concentrator for Solar Thermal Propulsion
}

\author{
Larry Leigh and Hamid Hamidzadeh \\ South Dakota State University \\ Mechanical Engineering Department \\ Brookings, South Dakota
}

\author{
Michael L. Tinker * \\ Structural Dynamics and Loads Group/ED21 \\ Structures, Mechanics, and Thermal Department \\ NASAMarshall Space Flight Center \\ Huntsville, AL 35812
}

\begin{abstract}
An inflatable structural system that is a technology demonstrator for solar thermal propulsion and other applications is characterized for structural dynamic behavior both experimentally and computationally. The inflatable structure is a pressurized assembly developed for use in orbit to support a Fresnel lens or inflatable lenticular element for focusing sunlight into a solar thermal rocket engine. When the engine temperature reaches a pre-set level, the propellant is injected into the engine, absorbs heat from an exchanger, and is expanded through the nozzle to produce thrust. The inflatable structure is a passively adaptive system in that a regulator and relief valve are utilized to maintain pressure within design limits during the full range of orbital conditions. Modeling and test activities are complicated by the fact that the polyimide film material used for construction of the inflatable is nonlinear, with modulus varying as a function of frequency, temperature, and level of excitation. Modal vibration testing and finite element modeling are described in detail in this paper. The test database is used for validation and modification of the model. This work is highly significant because of the current interest in inflatable structures for space application, and because of the difficulty in accurately modeling such systems.
\end{abstract}

\section{Introduction}

Solar thermal propulsion has received considerable attention in recent years as an economical means of enabling low-thrust orbital transfer and .perhaps deep space missions. The basic concept behind solar thermal propulsion is to utilize sunlight or solar energy as a means of heating a working fluid (propellant) to provide thrust at increased specific impulse. As described in Ref. 1, thrust is produced by expanding the heated propellant through a nozzle. No combustion occurs, and the thrust level is low. For this reason, solar thermal propulsive systems are mainly

\section{*Aerospace Technologist, Structural Dynamics; Associate Fellow AIAA}

Copyright $\theta 2001$ by the American Institute of Aeronautics and Astronautics, Inc. No copyright is asserted in the United States under Title 17, U.S. Code. The U.S. government has a royalty-free license to exercise all rights under the copyright claimed herein for Governmental purposes. All other rights are reserved by the copyright owner. applicable for orbital transfer vehicles and relatively longterm space missions.

The Shooting Star Experiment (SSE), for which the inflatable concentrator was developed, was designed as a precursor mission to the Solar Thermal Upper Stage (STUS), which is described in Ref. 2. The engine system for the STUS is designed to utilize hydrogen propellant to produce a thrust level of about 2 lbf. Two parabolic collectors will be used that can be rotated and gymballed for focusing sunlight into an absorber cavity (Fig. 1, from Ref. 3). The collectors will be inflated after separation of the upper stage from the launch vehicle.

The Shooting Star Experiment was intended to demonstrate the basic technologies required for the STUS, but on a smaller scale. Critical technologies developed or advanced for the SSE included not only the inflatable structure constructed of polyimide thin-film material, but also the polyimide lens, the thermal storage engine constructed of solid rhenium and rigidized rhenium foam, and a centering/pointing system. The focus of this paper is to (1) describe the inflatable and its subsystems, (2) discuss modal tests for characterizing the structure and the nonlinear polyimide film material, and (3) describe structural dynamic modeling of the inflatable based on test results.

\section{Description of the Inflatable Solar Concentrator Structure}

A major advantage of using inflatable structures in space is their extremely light weight. This makes inflatables an ideal match for solar thermal propulsion because of the low thrust levels available. An obvious second advantage is onorbit deployability and subsequent space savings in the launch configuration. A recent technology demonstrator flight for inflatable structures was the Inflatable Antenna Experiment (IAE) that was deployed on orbit from the Shuttle Orbiter. Although difficulty was encountered in the inflation/deployment phase, the flight was successful overall and provided valuable experience in the use of such structures (Ref. 4).

Initially, the SSE was designed to be carried on a Spartan spacecraft and launched in the Space Shuttle. In this design, an instrument canister would house the thermal engine and a secondary solar concentrator, and a special container would be used for storing the inflatable during launch. On orbit, the Spartan spacecraft and the SSE would be deployed using the Orbiter's remote manipulator arm. After release of the 
Spartan, pyrotechnics would open the inflatable structure container, with the inflation sequence following. Figure 2 shows conceptual details of the SSE in the on-orbit deployed configuration. The inflatable concentrator assembly consists of a thin Fresnel lens for focusing sunlight, a torus ring that supports the lens, and three struts that support the torus and attach to the spacecraft (Ref. 5).

On-orbit conditions make it necessary to provide an inflation system that can vary the pressure. As the spacecraft and inflatable structure moves from orbital eclipse into orbital day, the temperature of the nitrogen gas in the torus and struts could show a large increase, and consequently the pressure could increase. The relief valve must prevent the pressure from increasing above $1.0 \mathrm{psi}$ to prevent possible leakage or failure of the inflatable. Conversely, as the vehicle moves from orbital day into darkness, the pressure could drop drastically. The regulator must insure that the pressure does not fall below the desirable operating pressure (approximately $0.5 \mathrm{psi}$ ). The design pressure limits are driven by the strength of the various joints and seams in the inflatable torus and struts.

\section{Ground-Based Modal Vibration Testing for Development and Characterization of the Inflatable Structure}

A series of ground tests were performed to evaluate the performance of inflatable prototype structures for the Shooting Star Experiment. Modal survey, thermal shock, thermal static deflection, as well as limited deployment tests have been done to allow development of verified structural models and verify operation of the inflatable system. In this paper, the focus is on modal vibration testing in atmospheric conditions, and subsequent structural dynamic modeling and correlation with test.

Modal tests were performed for the prototype concentrator assembly in a vibration lab at atmospheric pressure (Ref. 6). It is realized that the air could have a considerable damping effect on the dynamics of the inflatable structures, and this was evaluated in a separate series of tests in both vacuum and ambient conditions, as described in Ref. 7. As shown in Fig. 3, the prototype concentrator in the test configuration consisted of an aluminum plate at the top of the structure, which represented the mounting fixture for the solar-thermal engine. Three tapered struts were aftached to the aluminum plate, with diameter varying from about 6.48 " at the top to 4 " in at the connection of the torus. The struts were approximately 72 " in length and were constructed of Kapton 100-HN film with an average thickness of 2 mils. The torus had a 6" diameter cross-section and outside overall diameter of 72" It was constructed from Kapton 300-JP film. The lens was simulated with a polyethylene sheet attached to the inner edge or flange of the torus.

The system was hung from three bungee cords for a "free-free" test. The pressure line is visible at the top of the structure, and a tank of pressurized air can be seen in the background. Excitation was provided with a shaker attached to the support plate at the top of the inflatable assembly. It was determined during pretest investigations that excitation could not be applied directly to the surface of any of the inflatable components to obtain acceptable response. Modal tests were run at three different inflation pressures, 0.25 , 0.50 , and $1.0 \mathrm{psig}$. As noted previously, $0.50 \mathrm{psi}$ is the desired on-orbit operating pressure.

During tests of the concentrator assembly, considerable leakage was observed, but it was concluded that the leak rate was slow enough to allow useful measurements. Considerable variation in the measured frequencies and mode shapes was observed for the three different pressures. Mode shapes, frequencies, and damping characteristics are described in detail in Ref. 6. It is planned to show some of these test results in the final paper.

\section{Structural Dynamic Modeling of the Inflatable Structure and Correlation with Test}

\section{Beam Element Modeling Approach}

The first approach was to model the structure using simple beam elements in MSC/Patran. The different mode shapes and frequencies were then found using MSC/Nastran, and these results were compared test data. Some effort was made to reflect the complexity of the joints and seams in the model in an effort to get better results. Also because of the lightweight nature of the structure, the model included added local masses to simulate the mass of the accelerometers. Also it was noticed by looking at the test data that there was significant coupling between the inflatable structure and the bungee suspension system. In the model an effort was made as well as including the interaction between the shaker and the test article. There were some correlation between the modal test data and the simplified beam element model for a few of the different mode shapes, but the model was unable to predict them all.

\section{Shell Element Modeling Technique}

The next step was to expand on the previous model by using shell elements to build up the structure in a more realistic manner. The shell element sizes were kept small, on the order of $0.5-1$ inch, and the total model had on the order of 20200 elements and 119508 degrees of freedom. This was done in an effort to have enough elements to observe the character of shell modes that were present, which the simple beam element model was unable to predict. It was thought that because of the model's ability to represent both beam-type and shell-type mode shapes that it would allow comparison with some of the test mode shapes that the beam model was unable to predict. Considerable effort was also put forth to realistically "inflate" the model, since it was known that pressure and the "pre-tensioning" of the films had significant impact on both the shell modes and beam modes. Results that were found from the pressurizing of the struts were very encouraging, along with full torus inflation.

An effort was made to apply as much detail to the two models as possible to closely match the real test article. Details such as the varying thickness and modulus of the glued joints, and added masses of the accelerometer, were incorporated. Also, flanges created by the extra material due to the joining of the torus halves and these flanges were also 
included in the models. A nonstructural mass element was incorporated to account for the weight of the pressurized air inside the structure. These refinements were done because it was found very early during development of the simplified beam model that including these seemingly minor details had the potential to change the behavior of the model significantly. This is due to the extremely lightweight flexible nature of the model.

\section{Beam Model Comparison to Test Results}

The beam-element model results were compared to test using Modal Assurance Criteria (MAC). It is seen in Table 1 that a few of the finite element model (FEM) mode shapes correlated very well to the test modes. Upon closer investigation, it was noticed that one mode shape in the FEM model had fairly high MAC numbers with a couple of different modes in the test data. The reasoning was that because of the limited number of measurement points, modes that "looked" very similar from a beam element standpoint were actually modes that also had shell activity. Because of the probability of the coupling of beam modes and shell modes, it was assumed this accounted in part for the lack of correlation between model and test, and led to development of the shell model.

\section{Shell Model Comparison to Test}

The scheme used to verify the shell model was to create part of the model and attempt to correlate it with previous test results, or other models, to validate the approach. Table 2 demonstrates such a validation. At this point the model (Fig. 4) was a suspended system consisting of the bungees, aluminum plate, and three pressurized struts. These tapered struts (as discussed earlier varying from 6.48" to 4" diameter) were compared to both data from a test article (that consisted of a 6" diameter non-tapered inflated strut) and to a FEM model of this 6" strut. The results were reasonably close, considering the differences in geometry of the two models and the test article, and were convincing that the model had very good potential for correlation to the full system test article.Full pressurization of the complete model (Fig. 5) was completed, but comparison and correlation with test data has not yet been completed. It is planned to include these results in the final paper.

\section{Summary and Conclusion}

Solar thermal propulsion is a concept for producing thrust sufficient for orbital transfers, and requires innovative, lightweight structures. This paper has presented a description of an inflatable concentrator that consists of a torus, Fresnel lens, and three tapered struts for mounting the inflatable to a spacecraft carrier. Ground-based modal vibration testing was discussed for characterization and verification of the solar concentrator assembly, and for providing a database for structural dynamic modeling. Finite element models of the concentrator were developed using both beam-element and shell-element approaches and compared to test data.

The models have very good potential to correlate with test data, if enough detail is observed in the creation of the model. Also, with further testing of inflatable structures, it will be necessary to increase the number of measurement points, so that shell modes can be distinguished from beam type mode shapes and incorporated into the comparison and validation of the FEM model. The results obtained were very encouraging, and indicate that further investigation and refinement of these models would lead to more realistic results.

\section{Acknowledgments}

Larry and Rodney Bradford of United Applied Technologies, Inc., have done innovative work in design and development of the inflatable concentrator assembly described in this paper using polyimide film materials. John Lassiter of the Dynamics Test Branch at Marshall Space Flight Center (MSFC) is acknowledged for his work in providing modal and frequency response test data used in this study.

\section{References}

1. Stewart, Jesse F., and Martin, James A.,"Dual Fuel Solar Thermal Stage: Ideal Analysis", Journal of Spacecraft and Rockets, Vol. 33, No. 5, 1996, pp. 752754.

2. Patel, S., and Emrich, W. J., Jr., "Solar Thermal Upper Stage (STUS) Feasibility Study", Proceedings of ASME/JSME/JSES International Solar Energy Conference, Maui, HI, March 1995.

3. Lyles, Garry M., "NASA Solar Thermal Propulsion Project", Solar Thermal Propulsion Workshop Proceedings, NASA Marshall Space Flight Center, Huntsville, AL March 19-20, 1997.

4. Freeland, Robert E., "Inflatable Antenna Flight Experiment Experiences", Solar Thermal Propulsion Workshop Proceedings, NASA Marshall Space Flight Center, Huntsville, AL March 19-20, 1997.

5. Shooting Star Experiment (SSE) Requirements, Verification and Compliance Document, MSFC-RQMT2630, July 1997.

6. Lassiter, John, "Shooting Star Experiment Prototype Inflatable Strut/Torus Assembly Modal Survey", ED73(97-69), NASA Marshall Space Flight Center, Huntsville, AL, June 1997.

7. Slade, Kara N., Tinker, Michael L., Lassiter, John O., and Engberg, Robert, "Comparison of Dynamic Characteristics for an Inflatable Solar Concentrator in Atmospheric and Thermal Vacuum Conditions", AIAA2000-1641, Proceedings of the 41st Structures, Structural Dynamics, and Materials Conference, April 3-7, 2000, Atlanta, GA. 


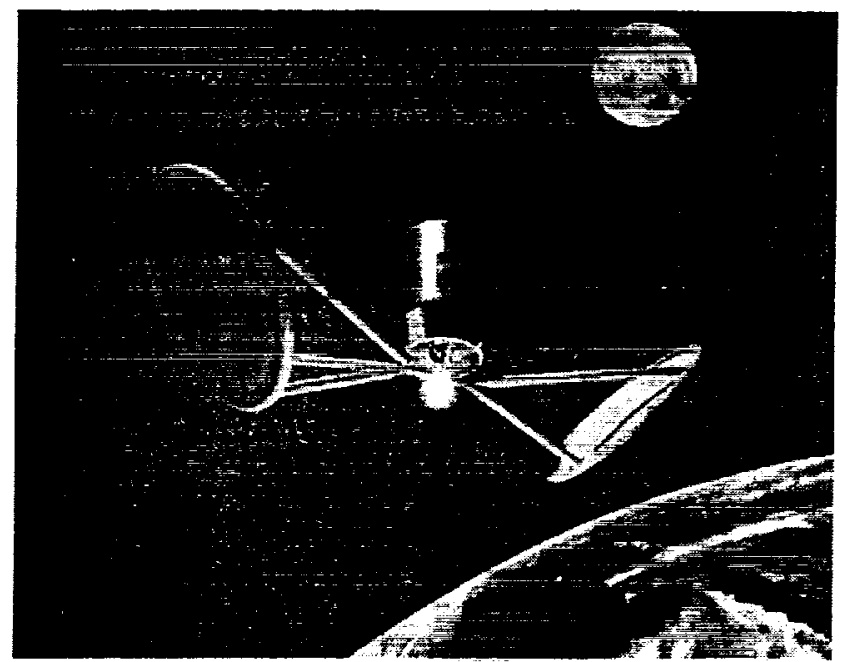

Figure 1. Solar Thermal Upper Stage Concept Utilizing Inflatable Concentrators

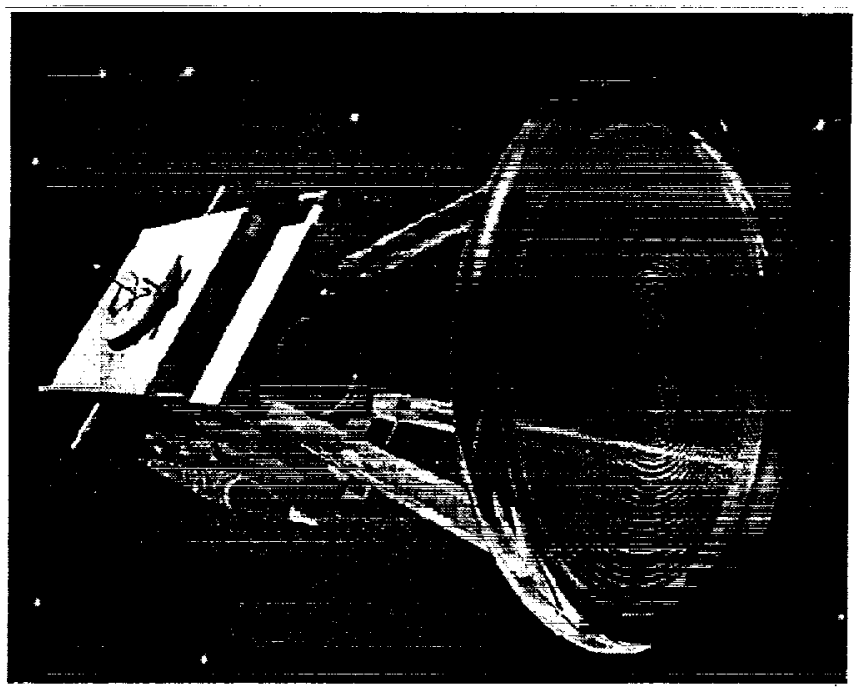

Figure 2. Concept for Shooting Star Experiment Solar Concentrator

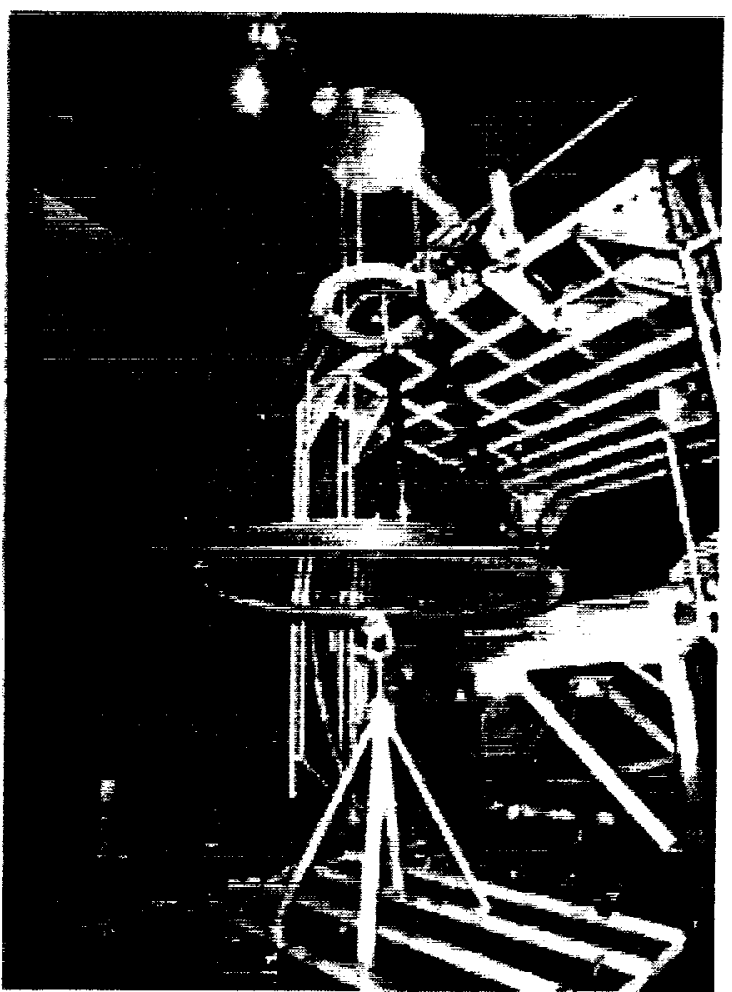

Figure 3. Shooting Star Experiment Prototype Concentrator with Free-Free Suspension System

Table 1. Initial Beam-Element Model Compared to Test Results for Concentrator Assembly

\begin{tabular}{|c|c|c|c|c|}
\hline $\begin{array}{l}\text { Test Model } \\
\text { Mode Number }\end{array}$ & $\begin{array}{c}\text { Test Model } \\
\text { Natural Frequency }\end{array}$ & $\begin{array}{l}\text { FEM Model } \\
\text { Mode Number }\end{array}$ & $\begin{array}{c}\text { FEM Modal } \\
\text { Natural Frequency }\end{array}$ & MAC Number \\
\hline 1 & 1.48 & 4 & 222.03 & 0.55 \\
\hline 3 & 6.29 & 7 & 1.82 & 0.62 \\
\hline 8 & 15.23 & 10 & 12.88 & 0.65 \\
\hline 9 & 17.19 & 14 & 32.17 & 0.59 \\
\hline 9 & 17,19 & 15 & 34.14 & 0.66 \\
\hline 9 & 17.19 & 44 & 222.03 & 0.61 \\
\hline 9 & 17.19 & 95 & 592.50 & 0.50 \\
\hline 10 & 26.76 & 100 & 652.89 & 0.55 \\
\hline 11 & 29.66 & 18 & 48.29 & 0.62 \\
\hline 11 & 29.66 & 19 & 48.88 & 0.61 \\
\hline 11 & 29.66 & 34 & 156.72 & 0.59 \\
\hline 11 & 29.66 & 100 & 652.89 & 0.75 \\
\hline 12 & 30.91 & 14 & 32,17 & 0.58 \\
\hline 12 & 30.91 & 15 & 34.14 & 0.55 \\
\hline
\end{tabular}


Table 2. Frequency Comparison for Current Model of Tapered Struts to Previous Untapered Model

\begin{tabular}{|l|c|c|}
\hline \multicolumn{3}{|c|}{ Cantilevered Beams } \\
\hline & Taper Model & Not tapered \\
\hline \multirow{2}{*}{$\begin{array}{l}\text { 1st Bending } \\
\text { 2nd Bending }\end{array}$} & 15.7 & 10 \\
\cline { 2 - 3 } & 66.1 & 53 \\
\hline
\end{tabular}

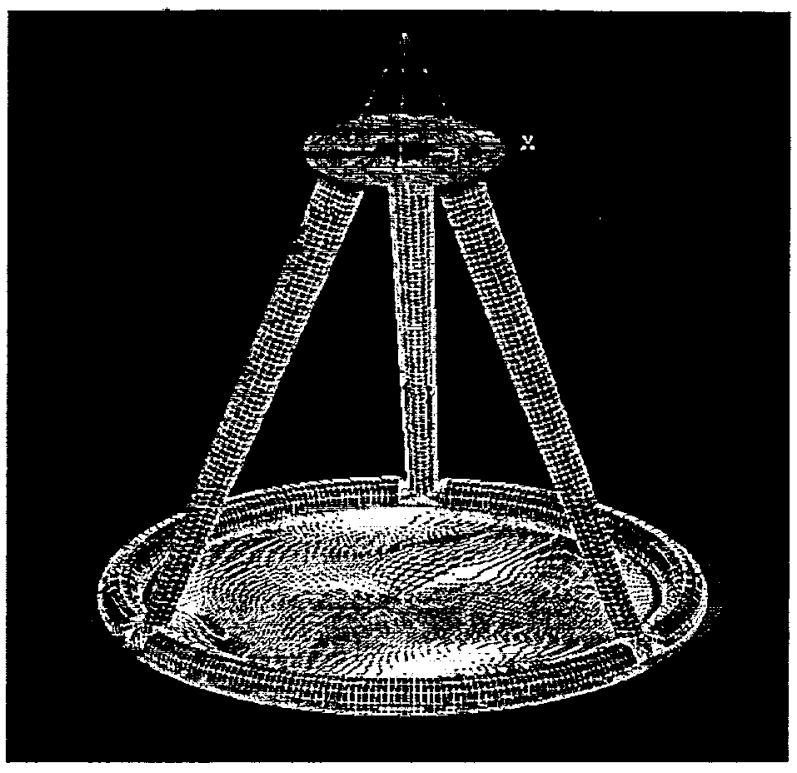

Figure 5. Full Model of Inflatable Concentrator

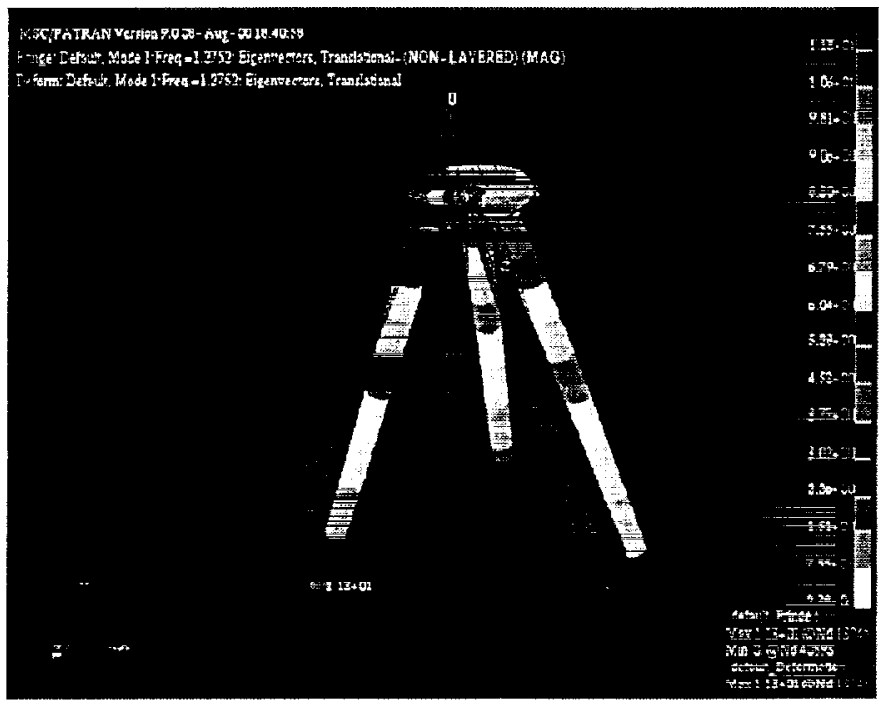

Figure 4. Model with Three Tapered Struts for Checkout Using Previous Test Data and Strut Models 


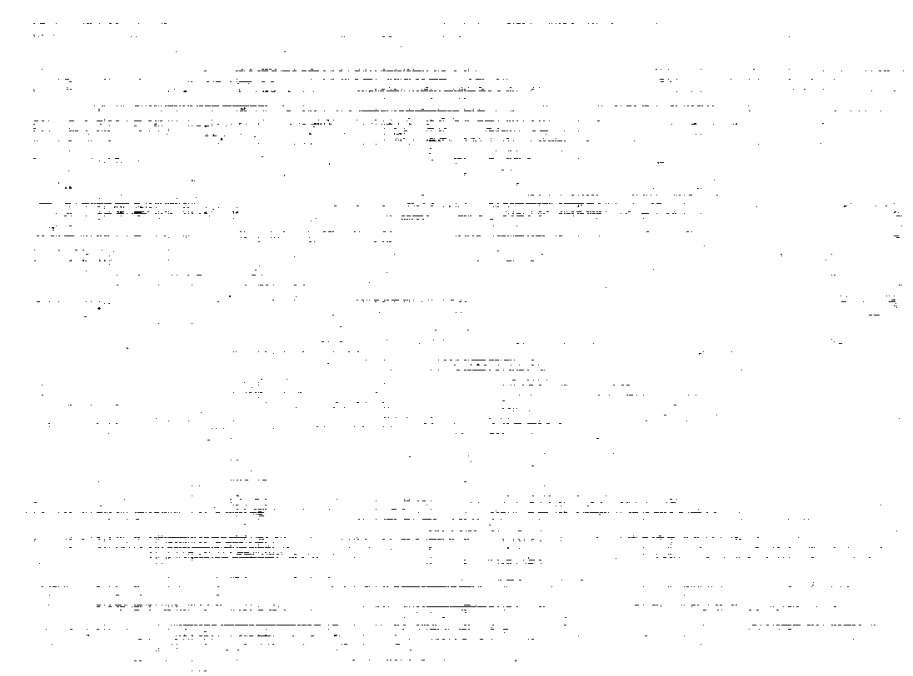

